\title{
Real Time Emissions Monitoring: The Foundation of a Blockchain Enabled Carbon Economy
}

\section{Nielsen $\mathrm{P}^{1,3 *}$, Johnston $\mathrm{S}^{2}$ and Black $\mathbf{P}^{2}$}

${ }^{1}$ Wood, Level 1, 240 St Georges Tce, Perth WA 6000, Australia

${ }^{2}$ Wood, Compass Point, 79-87 Kingston Rd, Staines TW18 1DT, United Kingdom

${ }^{3}$ Wood, 17325 Park Row, Houston, TX 77084, United States

*Corresponding author: Preben Nielsen, Wood, Level 1, 240 St Georges Tce, Perth WA 6000, Australia, Email: preben.nielsen@woodplc.com

\section{Review Article \\ Volume 5 Issue 2}

Received Date: May 31, 2021

Published Date: June 23, 2021

DOI: $10.23880 /$ ppej-16000265

\section{Abstract}

With $49 \%$ of the world's GDP under net zero goals, the global community is changing in how it treats emissions and carbon releases, with shareholders, stakeholders and investors demanding transparency on current performance and strategies to reduce or offset emissions. High frequency, reliable data empowers an organisation to strategically optimise and track emissions to reach committed goals from the asset level to the board room and across direct, indirect and supply chain sources (Scope 1, 2 and 3). A carbon footprinting solution, which provides a holistic view of total greenhouse gas emissions, requires a combination of carbon accounting, control system integration, emissions monitoring and greenhouse gas reporting software, to deliver an automated, reliable and verifiable real-time emissions/carbon reporting solution. This solution is also critical in providing managed data which can be utilised in the carbon economy and when combined with a Blockchain platform, results in a holistic data transfer chain for emissions reporting which is secure, transparent and trusted throughout industry and government. The role of comprehensive, connected environmental monitoring will be explored in the role of effective emissions offset and carbon trading economies with Blockchain supported technologies being presented as an enabling aspect of the overall solution. Smart contracts embedded within a Blockchain solution could automate trading mechanisms however require quality emissions monitoring data as a foundation for successful implementation. The role of quality emissions monitoring and governance in this process will be presented together with implications for industry and government for the carbon economy.

Keywords: Emissions; GHG; Greenhouse gas; Carbon; $\mathrm{CO}_{2}$; Blockchain; Smart contract; Energy transition; Sustainability; Net zero; Decarbonisation; Monitoring; Carbon price; Carbon trading; Regulator

\section{Introduction}

With increasing global consensus for a committed reduction to greenhouse gas emissions, governments and organisations are rapidly transitioning to new policies for the management of current and future emissions. Over
$49 \%$ of the world's GDP is under net zero emissions targets (ECIU 2020) [1] which is driving a concerted effort to deploy new technologies and strategies to demonstrate emissions reduction and offset. The energy industry has a significant role to play in overall decarbonisation with energy, heat 


\section{Petroleum \& Petrochemical Engineering Journal}

production and industrial processes accounting for over $55 \%$ of all global greenhouse gas emissions [2].

\section{Discussion}

\section{Emissions Monitoring}

A core requirement for the immediate and long-term vision of carbon reduction is the ability to thoroughly understand the real-time emissions from a facility, precinct or whole organisation. Only by ensuring accurate measurement can an organisation address the requirements for decarbonisation, make appropriate steps to reduce emissions and then validate the reduction against a baseline or starting point. Reliable and transparent emissions data is required for effective decision making throughout an organisation from the shopfloor to the boardroom.

Clear and accurate data is required to support both tactical decisions to reduce emissions during immediate day to day operations and inform strategic direction for long term initiatives. It is also important to have sufficient granularity of the data to assess emissions in temporal and spatial domains and over the range of emission origins. Reviewing past events informs current operations and allows real-time adjustment to operations whilst the prediction of future emissions due to planned changes informs the benefits of future initiatives. Similarly, the ability to differentiate emissions from specific locations in a plant or organisation may direct the attention to the largest emitting areas to achieve the greatest impact. Whilst much of the decarbonisation efforts currently underway focuses on Scope 1 (Direct) and Scope 2 (Indirect energy) emissions, significant attention is now being directed also to Scope 3 (Indirect supply chain) emissions. For a complete view on total emissions and the ability to measure progress against a decarbonisation roadmap a system should provide the ability to measure and report over all three scopes.

To gain a complete picture of a facility or organisational carbon footprint (the attributable total greenhouse gas emissions), a system must be designed and implemented to enable the automated aggregation, analysis and reporting of emissions related data and information. Collection of appropriate data is required from plant and enterprise systems such as data historians, enterprise asset management (EAP) and resource planning (ERP) systems, environmental management information systems (EMIS), relational databases and directly from the control system. Appropriate interfaces should be used to enable the reliability of data. The ability to perform aggregation, filtering, quality assessment and validation of the collected data in an automated fashion is a critical step to ensure high value information and enable the subsequent calculation and analysis for specific regulatory and organisational metrics. A key requirement for an environmental monitoring system is the reliability, security and auditability of the platform and the resulting reporting. Due to the regulatory requirements surrounding emissions collection and reporting, the ability to independently audit should be a fundamental requirement of a holistic system. With this functionality the system can deliver automated, reliable and auditable emissions data and analysis.

\section{Blockchain and Smart Contracts}

To support the aggregation and determination of carbon data, a secure means of transferring data and taking action on that information is required. Blockchain has been established as an effective means of ensuring private and immutable records. A distributed ledger complements an environmental monitoring solution to enable financial transactions that may be required under taxation and emissions trading schemes. A blockchain is a secure database consisting of a distributed ledger of data or transactions which is shared across a network of computers. Following validity checks from the network of computers, individual records are stored in a block or bundle of records which are then added to the end of the chain of previous blocks. The links between blocks are protected by a unique hash or encryption/identification code based on the contents of the block. This ensures the chain is connected in the correct order and protects the integrity of the chain as any change to an original single record will alter the hash and break the chain.

The various elements of a blockchain system align to provide the following key features; consensus: with the various node members combining to ensure the validity of each transaction, provenance: where the originator and destination and of each asset is marked, immutability: with the record of the distributed ledger being protected by the nature of the system, and finality: with all copies of the distributed ledger within the network holding the same record. A subsequent benefit for utilising a Blockchain solution for a supply chain financial system arises from the utilisation of smart contracts.

Smart contracts are computer programs running within in the Blockchain framework which utilise data within the chain to execute an agreement. The terms and details of the agreement are coded into the program which is then triggered by certain events or actions and a transaction is executed automatically. The actions taken by smart contracts are transparent across the entire network. Each party can independently verify the conditions and subsequent action taken. Because the data on the blockchain is immutable, smart contracts ensure trust between all parties involved in the transaction. 


\section{Petroleum \& Petrochemical Engineering Journal}

\section{Carbon Economy and Trading}

With a clear global consensus for the need to reduce emissions, many countries have initiated government led systems to encourage carbon reduction. There are many mechanisms for driving this behaviour in industry whilst still promoting economic growth. Most mechanisms rely on the ability to measure emissions and implement trading mechanisms to economically incentivise organisations to meet agreed emissions targets.

Whilst Australia does not currently have a nationally governed carbon price, the Australian Clean Energy Regulator oversees Australian carbon credit units (ACCUs) issued under the Emissions Reduction Fund and emission units issued under the Kyoto Protocol. These systems often suffer from high transaction costs, administrative complexity and the limitation of a secondary market for trading derivatives.

Despite the lack of formal pricing, many organisations are independently setting carbon prices for internal economic assessment and aligning carbon reduction targets to achieve net zero targets. Concurrently, there is a movement within investment houses to direct funds to organisations with a clear commitment to emissions reduction, providing further economic incentives to companies.

To enable a full cycle carbon economy from direct carbon pricing, emissions trading and secondary trading of economic derivatives, a robust means of carbon monitoring and accounting is required. Such a system provides not only the ability to reliably and accurately quantity emissions but also provides trust in the way transactions are administered through secure and auditable processes. The ability to automate the emissions data collection, analysis and reporting via an emissions monitoring system together with the security and immutability of a blockchain is a key enabler for aggregated regulatory reporting and carbon trading models.

Emissions monitoring systems for individual and private organisations are becoming more common globally. They provide a level of internal visibility to sources of emissions and aid in external reporting without risk of exposing confidential information as all data is held within internal networks. The information these systems generate is sent to independent and government supported regulatory bodies which gather information from multiple sites and entities. A monitoring system must ensure security of member organisations' data whilst enabling trust and external auditability of the aggregated data.

For these regulatory organisations, the transfer of sensitive emissions data from internal networks which have been collected using a trusted emissions monitoring system could be encrypted and transferred to the central body via a blockchain solution. Data can then be aggregated and analysed according to regulatory requirements to provide visibility over the broad emissions values based on a mutually trusted system without revealing sensitive corporate data.

The utilisation of blockchain for carbon trading has been explored in the context of a decentralised methodology which reduces the entry thresholds for carbon trading $[3,4]$. However while providing a framework for blockchain enabled trading there remains a requirement for robust emissions data to drive this mechanism.

A combined emission monitoring and blockchain enabled solution can be extended to enable carbon trading via the use of smart contracts. In such a system a partially decentralised blockchain could be utilised by a regulator with the power to issue and verify carbon credits and administer transactions. The system could utilise smart contracts that would automate the issuing of carbon credits as a direct emission reduction thresholds were met or offsetting activities such as carbon sequestration or reforestation occurred. This system provides an independent administration over carbon trading with transparency over the transactions. By utilising a connected monitoring system, the emissions data can be utilised and actioned in real-time, increasing the efficiency of the system and ensuring the accuracy of reported values.

Some key limiting factors and barriers to adoption for emissions driven, blockchain enabled trading include the rapid pace of change in technologies, a proliferation of solution vendors, limited interoperability between blockchains, a lack of a standardised smart contract library and uncertainty in legal enforcement of smart contracts.

\section{Conclusion}

A comprehensive system as described above would enable an efficient, transparent system with inherent security and integrity through the auditability of a complete emissions monitoring and reporting system and the immutability of a blockchain system.

To achieve this, a regulatory organisation for a particular industry or the overall clean energy regulator would have to align on a system for data gathering and transaction with invested industry bodies and companies. The regulatory body, backed by the government, and together with the member organisations should transition to this system over a period which allows for the implementation of automated data collection and validation against existing methods to ensure confidence in the system and demonstration of the transaction methods. 


\section{Petroleum \& Petrochemical Engineering Journal}

The key drivers for this system will be accelerated by global adoption of similar systems and the establishment of an emissions trading framework within Australia, including a specific carbon pricing mechanism. By engaging the free market, overall sector and country outcomes could be achieved with significant efficiency whilst ensuring regulatory oversight and auditability.

\section{References}

1. Energy \& Climate Intelligence Unit (ECIU) (2020) Almost half of global GDP under actual or intended net zero emission targets.

2. Edenhofer O, Pichs-Madruga R, Sokona Y, Farahani E,
Kadner S, et al. (2014) Climate Change 2014: Mitigation of Climate Change, Working Group III Contribution to the Fifth Assessment Report of the Intergovernmental Panel on Climate Change. Intergovernmental Panel on Climate Change (IPCC), Geneva, Switzerland.

3. Pan Y, Zhang X, Wang Y, Zhou S, Li G, et al. (2019) Application of Blockchain in Carbon Trading. Energy Procedia 158: 4286-4291.

4. Liss F (2018) Blockchain and the EU ETS: An architecture and a prototype of a decentralized emission trading system based on smart contracts. Thesis, Technische Universität München, Germany. 